previonsly and had been sent in by her medical attendant for operation. Her temperature was $102^{\circ} \mathrm{F}$. ; the pulse was 110 ; she had romited several times during the last 24 hours and looked distressed and ill. The abdomen was distended but moved slightly and she had a definite easily demonstrable " rectus segment rigidity." She was at once removed to the theatre and under chloroform an incision four inches long was made in the direction of the external oblique fibres starting from a point three-quarters of an inch internal to the anterior iliac spine and carried downwards and inwards about an inch above the level of Poupart's ligament. Aponeurosis and muscle fibres were separated in "gridiron" fashion and the peritoneum was opened at its outermost limit. The finger was passed back to the cæcum and was then carried downwards and almost at once struck foul, thin pus, which was mopped up as it welled up from the pelvis. The uterine appendages presented and were of a deep purple colour. Then the appendix was found adherent to the posterior aspect of the cæcum and it was freed and delivered with a minimum of manipulation; the mesoappendix was tied off and a catgut ligature was put round the base of the appendix, which was then cut away. The pelvis, \&c., were now gently but thoroughly swabbed dry and a thick gauze drain packed down into the abscess cavity (there was only a very inefficient attempt at shutting off the pus by adhesions). The gridiron incision was closed in layers except where the gauze protruded and the patient was propped up in bed (to prevent pus tracking up) and kept on hot water only. In 48 hours ethyl chloride was given and the gauze was removed and a fine gauze wick was put just inside the peritoneum. This was left out altogether after another two days. The temperature was normal on the third day and the pulse was 76 and flatus had been freely passed.

The incision is planned to get as far away from the general peritoneal cavity as possible and the cæcum is got at at once in this way. I have only twice found the gridiron incision too limited; in one case (a huge appendix pointing to the umbilicus) the oblique and transverse muscle fibres were separated up to the rectus sheath, which was divided vertically, giving lots of room and union of undivided muscle afterwards; in the other case there was very diffused pus and the original incision was converted into that described as Abernethy's for tying the internal iliac artery and free swabbing of pelvis and lower abdomen generally made possible. That was two years ago and the girl has a firm scar now. But whether the incision be made throngh the rectus sheath or close to Poupart's ligament, whether a gauze drain be used or a split rubber tube, whether rectal irrigation be utilised or the patient encouraged to drink hot water copiously, if the perforated appendix and its surrounding pus are cleared away sufficiently early Nature will set to work to form adhesions and limit the infection and in a most surprisingly short time the patient will be out of danger.

Bradford.

\section{ON SOME MODERN METHODS FOR THE CLINICAL EXAMINATION OF URINE AND GASTRIC JUICE BY THE GENERAL PRACTITIONER.}

Bx C. F. R. WEISS, M.A., Ph.D., F.C.S., M.P.S.

NowADAYS, in every branch of medicine, the great importance of accurate clinical investigation is well recognised. While, however, clinical laboratory investigation is pursued as a matter of routine in almost all the medical institutions of the world and also by many private practitioners, yet, owing to the tedious nature of many of the processes and the large amount of time necessary for their performance, they are wholly or in part omitted by the vast majority of medical men. Perhaps the factor most responsible for this defection is the unsuitability of the methods in vogue in the hospitals and large laboratories to the capacities of the private worker. A laboratory assistant who makes quantitative estimations in anything from five to 20 specimens is able to employ the necessary time to far better purpose than the isolated practitioner who is working on a solitary specimen, even though the examination may be the one thing needed to assist him in arriving at a proper conclusion with regard to his case. Yet the isolated worker stands in just as much need of assistance of this kind in arriving at a diagnosis as the hospital resident with his well-equipped laboratory.

1t is the purpose of the present paper briefly to pass in review certain of the newer methods for the quantitative examination of both urine and gastric juice which have but recently filtered across to this country, and to show that in addition to other merits they possess that of being well within the scope of the daily routine of the general practitioner.

Let us first take the case of albumin in the urine. I wonder were a census on the point to be taken how many general practitioners would be found to possess the ordinary albuminometer of Esbach, and, even possessing the instrument, how many use it with anything approaching habit? Until quite recently, with the exception of the Purdy tube, this was practically the only piece of apparatus by means of which the amount of albumin present in any sample of urine might be determined with reasonable facility. Doubtless much useful work has been done with Esbach's tube, but it is quite certain that a test which is only accurate for values at or above one-half pro mille, and which besides takes 24 hours to complete, falls very far short of the ideal. A much more efficient instrument ${ }^{1}$ has lately appeared and is described by its inventor, Dr. H. R. Harrower, in the Jnumal of the American Medical Association (Vol. LI., No. 7) It consists of a graduated tube and a reagent (phosphotungstic acid, 1.5 grammes; concentrated hydrochloric acid, five grammes; and 95 per cent. alcohol, 100 cubic centimetres). One cubic centimetre of urine is diluted with nine cnbic centimetres of distilled water and this is added drop by drop to the reagent, shaking after each addition until the appearance of a faint white cloud. This denotes the completion of the reaction and the level of the fluid in the tube is read off. It corresponds to the amount of diluted urine which contains one-tenth of a milligramme $(0.0001$ gramme) of albumin. The percentage is easily reduced but a table is supplied with the instrument to assist the calculation. This is without doubt a far more practical instrument for both hospital assistant and home worker than the Esbach or Purdy tubes, and some idea of the accuracy of the test may be gathered from the fact that it is sensitive to one-tenth of a milligramme.

In the matter of the estimation of sugar also much has been done in the later instruments to simplify and to facilitate the test without impairment of accuracy. While Fehling's solution is undoubtedly one of the best reagents for qualitative work, its application to quantitative analysis in the hands of the inexperienced is fraught with serious jeopardy to accurate work. When one remembers with what apparatus and under what conditions of light, \&sc., these estimations are often made in the surgery of the general practitioner, one can but dimly speculate upon the possibility of anything in the shape of a reliable result being forthcoming. Moreover, apart from the possibility of experimental error, there are actual fallacies attendant upon the use of Fehling's solution. These are due to the fact that other substances in the urine can reduce cupric oxide. The chief of these are uric acid, creatinine, hippuric acid, lactose, glycuronic and glycosuric acids, and certain drugs. There is only one absolutely certain test for glucose-i.e., the fermentation test, glucose being the only fermentable substance ever found in urine. Much attention has lately been paid to the improvement of the various forms of saccharometer-i.e., instruments for estimating the amount of sugar by the fermentation method. The latest instrument of this kind, which will be found to fulfil every requirement in practice, is a graduated $\mathrm{U}$ tube in which evolution of carbon dioxide by fermentation causes displacement of a column of mercury. It is possible to register the sugar contents in twentieths per cent. up to 1.5 per cent. and thence in halves per cent. to 10 per cent. The instrument is cheap, simple, and effective, and will doubtless supersede all other forms of saccharometer.

For the estimation of urea no better piece of apparatus has been devised than that of Doremus. The improved form of

1 This and all the apparatus described in the paper can be obtained from all purveyors of chemical and medical apparatus. The "Qualitest" (price 16s.6d.) and "Quantitest" (price 33s. per case). 
this excellent instrument renders possible the urea determination in 1 cubic centimetre of urine within the space of a few minutes. The only reagents required to be kept at hand are a 40 per cent. solution of sodium hydrate and measured quantities of bromine contained in bulbs.

The estimation of these three-albumin, sugar, and urearepresents the extent to which urine is chemically examined for clinical purposes by anyone not in possession of a clinical Taboratory. It has hitherto been impracticable, for instance, for the average practitioner to determine the contents in a given specimen of urine of so important a constituent as uric acid. He takes up his text-book in search of an available method and finds himself duly instructed in the details of such procedures as that of Hopkins. What he is not told is that, ready to hand at a minute's notice, is a reliable instrument such as that of Ruhemann, by means of which the uric acid mystery may be solved within the space of half an hour or less. I do not think this method is very generally known in this country; its importance certainly entitles it to some description here. The principle involved is the fixation of free iodine by uric acid. The apparatus consists of a graduated tube and two reagents-(1) an iodo-iodide solution resembling Gram's solution in composition, and (2) bisulphide of carbon. The latter is poured into the tube up to the mark indicated; the iodine solution is added to a further mark. Diluted urine is then slowly added until the bisulphide of carbon, which from a deep violet hue acquired on the first addition of urine, passes through various depths of pink and finally becomes snow-white. This denotes the completion of the reaction and the level of the mixture in the tube is read off on a graduated scale provided and indicates in values pro mille the amount of uric acid present in the diluted urine. The possibilities of this simple test in private practice are too important to be overlooked. The apparatus is quite inexpensive and its utility will well repay the outlay.

Another simple quantitative test is that for phosphates. This is done by a reliable little instrument called a "phosphatometer." It consists of a graduated tube and one reagent. The tube is so graduated that all that is necessary is to add the reagent to a given quantity of urine and read off the level of the resultant precipitated phosphates on the scale provided. The reagent in this case consists of an ammonio-magnesium mixture composed as follows : magnesium chloride, five grammes; ammonium chloride, seven grammes; strong solution of ammonia, 35 grammes; and distilled water, 65 grammes. The phosphates are precipitated by this mixture in the form of the ammonio-magnesium variety. A table is provided with the instrument for calculating percentages.

Quantitative tests as simple and as effective as the foregoing exist also for the determination of indican and total acidity or alkalinity. Space forbids their detailed description here, but in their respective channels they may be made to figure usefully in a well-arranged scheme of accurate diagnosis.

Passing now from the subject of urine to that of gastric juice we find that in this field also chemical industry has been active in providing the home worker with similar means of diagnosing conditions of complexity which bafle other sources of information. The modern medical man is taught the importance of the rôle played by hydrochloric acid in gastric digestion, that its diminution or absence suggests one chain of thought, its hypersecretion another chain. Of what use is this knowledge to him in the absence of means of ascertaining which condition he is dealing with ? In the present state of his resources it is only by prolonged observation accompanied by much empirical treatment with acids and alkalies that he may at length arrive at a rough estimate of the amount of hydrochloric acid present in the stomach of his patient. Wherein does the remedy lie ? Clearly in submitting the gastric juice itself to chemical analysis whereby its acidity may be determined. Here, again, his outfit has been woefully lacking in provision for this important step towards benefiting a very large class of his clientèle. An instrument is now to be had whereby may be made three important quantitative tests-viz., the estimation of free hydrochloric acid, total acidity, and combined hydrochloric acid. These results are all obtained by the use of a graduated tube devised by Gunzberg, and, together with a few physical tests very easily performed, give all the information which is required on the subject.
The instrument, termed an " acidimeter," is a simple graduated tube and in using it two reagents are required: (1) decinormal solution of sodium hydrate and (2) an indicator consisting of phenolphthalein and dimethyl-amidoazobenzol (one gramme of each) and 70 per cent. alcohol 100 cubic centimetres. Gastric juice is obtained by means of a syphon tube after a test meal and filtered through ordinary filter paper. This is poured into the tube to a given mark and the addition of two drops of the indicator solution gives a bright cherry-red colour. The decinormal soda is now added drop by drop until the colour of the fluid has changed from red to a distinct canary-yellow. The level is then read off on a scale (marked red) which indicates without any calculation the percentage of free hydrochloric acid present. This being noted down, the test is continued with the same solution by dropping in more sodium hydrate until the fluid has become permanently red. The level as indicated by another scale (marked yellow) gives the total acidity. Should there be no free hydrochloric acid present, a dirty yellow colour will result on adding the indicator. To find the amount of combined hydrochloric acid all that is necessary is to repeat the previous test using as an indicator a 1 per cent. aqueous solution of sodium alizarin-sulphonic acid. The decinormal soda is added drop by drop as before until a deep violet colour appears. The yellow figures subtracted from the previously determined total acidity furnish the required result, for the total hydrochloric acid is equal to the sum of the free and combined acid.

Thus, it will be seen that information of the utmost importance from a diagnostic point of view may be gained in a comparatively short space of time and much experimental therapy done away with. Moreover, the assistance rendered by instruments such as these goes far towards placing the medicine of the general practitioner upon the same firm basis as that of the hospital or clinic to which too often he is compelled to refer his more complex cases.

Harp-lane, E.C.

\section{A NEW OPERATING TABLE.}

\section{BY ERNEST W. HEY GROVES, M.D., M.S. LOND.,} F.R.C.S. ENG.,

ASSISTANT SURGFON TO THE BRISTOL GRNERAL HOSPITAL AND SURGEON TO THE COSSHAM MEMORIAL HOSPITAY

AND

E. H. E. STACK, M.B., B.C. CANTAB., F.R.C.S. ENG, ASSISTANT SURGEON TO THE BRISTOL ROYAL INFIRMARY AND SURGEON TO THE COSSHAM MEMORIAL HOSPITAL.

WHEN we were recently asked by the trustees of the late Mr. Handel Cossham to advise them as to the best surgical equipment of an up-to-date hospital we found that the choice of an operating table presented no little difficulty. Most of the English models were made on four legs with or without casters, and all of them which could be raised or lowered were actuated by hand levers only, which necessitated an arduous and laborious task, and one which sometimes means the disturbance of the operation and re-arrangement of the patient. Most of the newer continental makers have adopted the central single pillar support and a few have introduced the foot-operated oil pump for raising purposes. Most of these models seemed to us to be of a somewhat unsteady type and the more elaborate--e.g., Doyen's-table is undesirably intricate besides costing 100 guineas. We therefore designed a table which would combine facility of manipulation with strength, stability, and simplicity, and after submitting the designs to an engineer and laying the same before the trustees we were instructed by the latter to have the table constructed for the Cossham Memorial Hospital. It has now been in use for over a year for every variety of operation and it appears to us to fulfil the objects of its design, and we therefore venture to describe it in the hope that it may prove of use to others.

Support. - The table is supported on a centrally placed vertical steel bar which forms part of the piston of a gunmetal cylinder imbedded 18 inches deep into the floor of the operating theatre.

Raising and lowering.-By the admission of water at a pressure of 20 pounds to the square inch into the cylinder, 